\section{Avaliação de reprodutibilidade e validade de um questionário de freqüência alimentar em adultos residentes em Porto Alegre, Rio Grande do Sul, Brasil}

\author{
Assessment of reproducibility and validity of a \\ food frequency questionnaire in a sample of adults \\ living in Porto Alegre, Rio Grande do Sul State, \\ Brazil
}

\author{
1 Programa de Pós-gradu- \\ ação em Saúde Coletiva, \\ Universidade do Vale do Rio \\ dos Sinos, São Leopoldo, \\ Brasil. \\ 2 Faculdade de Nutrição, \\ Universidade Federal \\ Fluminense, Niterói, Brasil. \\ 3 Programa de Pós-gradu- \\ ação em Ciências Médicas, \\ Universidade Federal \\ Fluminense, Niterói, Brasil. \\ Correspondência \\ M. T. A. Olinto \\ Programa de Pós-gradu- \\ ação em Saúde Coletiva \\ Universidade do Vale do \\ Rio dos Sinos. \\ Av. Unisinos 950, São \\ Leopoldo, RS \\ 93022-000, Brasil. \\ mtolinto@unisinos.br
}

\begin{abstract}
The reproducibility and validity of a Food Frequency Questionnaire (FFQ) was evaluated in 83 adults living in a State capital in southern Brazil. The reproducibility and validity were tested with intra-class correlation coefficients (ICC). Mean nutrient intakes from three 24-hour dietary recalls (24hDR) were compared to mean intakes from each FFQ (FFQ1 and FFQ2), adjusted for energy intake and within-person variation. $\mathrm{Nu}$ trients were categorized into quartiles and assessed using weighted kappa statistics. Mean ICC for the test-retest FFQ ranged from 0.44 (95\%CI: -0.19-0.76) to 0.83 (95\%CI: 0.72-0.90) for energyadjusted carbohydrate and vitamin $C$ intakes, respectively. Mean ICC, energy-adjusted and corrected for within-person variability, ranged from 0.23 (95\%CI: -0.22-0.61) to 0.86 (95\%CI: 0.21 1.16) for vitamin $A$ and lipids for the FFQ1, and 0.50 (95\%CI: -0.16-0.91) to 0.95 (95\%CI: 0.791.05) for vitamin A and energy for the FFQ2. For the FFQ1, calcium showed the lowest weighted kappa value (0.10; 95\%CI: 0.00-0.26), while vitamin C (0.38; 95\%CI: 0.23-0.53) and total energy (0.38; 95\%CI: 0.24-0.52) had the highest weighted kappa for the FFQ2. The FFQ showed good reproducibility and reasonable relative validity.
\end{abstract}

Questionnaires; Feeding Behavior; Adult
Anelise Fernanda Zanolla 1

Maria Teresa Anselmo Olinto 1

Ruth Liane Henn 1

Vivian Wahrlich 2

Luiz Antonio dos Anjos 2,3

\section{Introdução}

A Organização Mundial da Saúde situa o padrão alimentar entre as principais causas de doenças não-transmissíveis e mortalidade. Por outro lado, nos últimos anos, estudos epidemiológicos de base populacional e ensaios clínicos randomizados evidenciam o papel da dieta na prevenção e controle da morbimortalidade devido a essas doenças 1,2 .

Diante de tais constatações, fica clara a necessidade de investigar o padrão de ingestão alimentar para se compreender de que forma a dieta impacta o processo saúde-doença. A avaliação da ingestão alimentar exige metodologia padronizada, com o uso de instrumentos válidos, reprodutíveis e confiáveis 3,4 .

Entre os métodos de avaliação mais utilizados destacam-se o diário alimentar (DA), o inquérito recordatório de 24 horas (IR24h) e o Questionário de Freqüência Alimentar (QFA). Enquanto os dois primeiros podem ser aplicados em qualquer população, sem necessidade de validação, o QFA precisa ser validado na população objeto de estudo, uma vez que mudanças sutis nos alimentos que o compõem podem afetar o seu desempenho ${ }^{3}$. Além disso, diferentes grupos demográficos e culturais exigem questionários de freqüência alimentar que considere as suas especificidades de alimentação 5 . Um instrumento válido indica que a informação mensurada reflete exatamente o que ele pretende medir. 
A necessidade do uso do QFA em populações intensificou a realização de estudos de validação deste instrumento, comparando-o a outros métodos 6 . O IR24h tem sido o método de referência mais utilizado, especialmente quando a população apresenta baixo grau de escolaridade ou pouca participação e motivação 1. Para investigar a validade de um QFA é necessária a comparação com a média de, no mínimo, duas medidas feitas com o método de referência7. No entanto, quando se utiliza um pequeno número de replicatas, recomenda-se um ajuste estatístico para a remoção dos efeitos de variação intrapessoal 8 . Além da validade, a reprodutibilidade também é necessária para atestar qualidade a um instrumento. Reprodutibilidade mede se os resultados são similares em duas ocasiões distintas de mensuração ${ }^{9}$.

O presente estudo teve como objetivo medir a reprodutibilidade e a validade relativa de um QFA contendo 127 itens alimentares, em uma amostra de adultos residentes na região metropolitana de Porto Alegre, Rio Grande do Sul, Brasil.

\section{Métodos}

O estudo foi realizado em uma amostra, obtida por conveniência, de indivíduos de 20 a 69 anos de idade, residentes na região metropolitana de Porto Alegre. Para a seleção dos participantes fezse a divulgação em um jornal de grande circulação do município e em cartazes espalhados por diversos pontos da região. Entretanto, como a coleta de dados ocorreu no centro clínico de um hospital privado de Porto Alegre, a maioria dos indivíduos selecionados constituiu-se de funcionários e usuários da referida instituição.

Inicialmente, recrutaram-se 113 indivíduos que concordaram em participar do estudo. Desses, 17 (15\%) realizaram somente a primeira entrevista e $13(11,5 \%)$ a primeira e segunda entrevistas, resultando em uma amostra de 83 pessoas (52 mulheres) para as análises.

Para fazer parte da amostra as pessoas deveriam possuir, no mínimo, a 4a a série do Ensino Fundamental. Os critérios de exclusão foram: apresentar diabetes mellitus, doenças renais, doenças gastrointestinais, ou qualquer outra patologia ou sintoma que interferisse no hábito alimentar, e não estar em dieta orientada por nutricionista ou endocrinologista. Para as mulheres, estar grávida no momento da seleção também foi critério de exclusão.

Os instrumentos utilizados foram questionários sócio-econômico e demográfico (padronizados, pré-codificados e pré-testados), QFA e IR24h. Cada participante foi entrevistado em três momentos por entrevistadores treinados. A duração média do estudo foi de 150 dias, entre os meses de agosto e dezembro de 2006.

\section{Questionário de Freqüência Alimentar}

Este estudo constitui-se em uma etapa preliminar do projeto PROCAD 01/2005, nº. 0257052, desenvolvido pelo Programa de Pós-graduação em Saúde Coletiva da Universidade doVale do Rio dos Sinos (UNISINOS), Universidade Federal Fluminense (UFF) e Escola Nacional de Saúde Pública Sergio Arouca da Fundação Oswaldo Cruz (ENSP/ FIOCRUZ), que tem por objetivo construir um instrumento para avaliar o consumo alimentar de população adulta. Sendo assim, a lista de alimentos do QFA foi construída a partir das informações obtidas com a aplicação de IR24h em uma amostra probabilística de 1.726 adultos avaliados na Pesquisa de Nutrição, Atividade Física e Saúde (PNAFS), inquérito domiciliar realizado em adultos residentes em Niterói, Rio de Janeiro 10. Todos os alimentos citados nos IR24hs por, no mínimo, 30 indivíduos, foram incluídos no questionário. Alguns alimentos foram agrupados segundo suas características nutricionais, o que resultou em uma lista com 127 itens alimentares (QFA-127). Esse questionário previa, ainda, um espaço para que os entrevistados relatassem alimentos não previstos na lista original, mas que eram característicos do padrão alimentar do Sul do Brasil. Quanto à freqüência, os participantes informavam se era semanal ou mensal e o número de vezes ao dia que cada um dos 127 itens alimentares foi consumido no mês anterior à entrevista. Esse questionário foi do tipo quantitativo, em que os participantes dimensionavam o tamanho da porção usualmente consumida com a ajuda de um álbum fotográfico 11 . O QFA foi aplicado na primeira e na última entrevistas, com um intervalo médio de 28 dias.

\section{Inquérito recordatório 24 horas}

O IR24h avaliou o consumo de alimentos e bebidas ingeridos nas 24 horas anteriores à entrevista. Com a ajuda do álbum fotográfico, o participante relatava, detalhadamente, sobre o tamanho e volume da porção consumida, marcas dos produtos e modo de preparação. Os IR24hs foram aplicados durante três entrevistas, com um intervalo médio de 14 dias, sendo que um deles, obrigatoriamente, era aplicado na segunda-feira, para que fosse coletada a ingestão alimentar do domingo.

As informações obtidas pelos IR24hs e QFAs foram analisadas pelo programa Nut Win, versão 1.5.2.51 (Programa de Apoio à Nutrição, desen- 
volvido pelo Departamento de Informática em Saúde, Universidade Federal de São Paulo, São Paulo, Brasil). Alguns alimentos não faziam parte do banco de dados do programa e foram inseridos no mesmo. Para tanto, utilizaram-se informações da TACO (Tabela Brasileira de Composição de Alimentos) 12, da Tabela para Avaliação de Consumo Alimentar em Medidas Caseiras 13 e dos rótulos dos alimentos. Enquanto os dados dos IR24hs foram digitados diretamente no programa Nut Win, para os QFAs houve uma etapa prévia de transformação da ingestão alimentar em gramas ou mililitros/dia. Isso foi feito com o auxílio de uma planilha de Excel (Microsoft Corp., Estados Unidos), multiplicando-se a freqüência pelo tamanho da porção informada e dividindo este produto pelo número de dias no mês. Para cada um dos IR24hs e dos QFAs determinou-se o valor energético total, bem como de proteína, lipídio, carboidrato, cálcio e vitaminas A e C.

\section{Análises estatísticas}

Todas as informações foram transferidas para o SPSS, versão 11.0 para Windows (SPSS Corp., Chicago, Estados Unidos), inclusive aquelas dos questionários sócio-econômico e demográfico.

Média, desvio-padrão e diferença entre as médias foram calculados para valores absolutos de energia e demais nutrientes ingeridos nos dois QFAs e no IR24h. Diferenças entre as médias foram verificadas com teste t pareado.

Para as demais análises, energia e nutrientes de ambos os métodos foram log transformados, a fim de normalizar a distribuição dos dados.

A correção pela energia foi feita computando-se os resíduos de modelos de regressão, com a ingestão energética como variável independente e a ingestão dos nutrientes como variável dependente 14

Para verificar a reprodutibilidade do QFA, calculou-se o coeficiente de correlação intraclasse (ICC) por ponto e por intervalo de $95 \%$ de confiança (IC95\%).

A validade relativa foi avaliada por meio dos ICC entre os nutrientes brutos e corrigidos pela energia, por ponto e por IC95\%. Devido à atenuação causada pela variação dia-a-dia na ingestão alimentar intra-sujeito, os coeficientes foram corrigidos pela razão das variâncias intra e entre sujeitos nos três IR24hs, com a seguinte equação:

$$
\mathrm{r}_{\mathrm{v}}=\mathrm{r}_{\mathrm{o}}(1+\lambda / \mathrm{n}) 1 / 2
$$

em que $r_{\nu}$ é a correlação verdadeira, $r_{o}$ a correlação observada entre o QFA e a média dos IR24hs, $\lambda$ é a razão da variância intra e entre sujeitos nos IR24hs e $n$ é o número de replicatas, neste caso, três recordatórios. Coeficientes de correlação en- tre 0,4 e 0,7 indicam boa concordância entre os dois métodos de avaliação da dieta 15 .

A concordância entre cada QFA e a média dos três IR24hs também foi avaliada por meio da classificação dos indivíduos segundo as distribuições dos participantes em quartos de consumo de energia e demais nutrientes, de cada método. Os percentuais de concordância exata (classificação no mesmo quarto por ambos os métodos) e de discordância (classificação em quartos opostos) foram estimados. Para a análise, a classificação em quartos foi comparada por meio do índice kappa ponderado.

Todos os procedimentos para avaliar a reprodutibilidade e a validade do QFA também foram testados na amostra estratificada por sexo.

Este estudo foi aprovado pelo Comitê de Ética em Pesquisa da UNISINOS. Após esclarecimentos, os participantes que aceitaram participar da pesquisa assinaram o Termo de Consentimento Livre e Pré-informado, ficando assegurada a confidencialidade das informações. Todos foram comunicados que poderiam retirar-se do estudo a qualquer momento.

\section{Resultados}

Os participantes tinham, em média, 41 anos de idade (desvio padrão - DP $\pm 13,36)$ e 13 de escolaridade ( $\mathrm{DP} \pm 3,42)$. Cinqüenta por cento dos entrevistados eram casados e $71 \%$ estavam empregados. Entre os indivíduos que não finalizaram o estudo, 50\% eram mulheres, tinham, em média, 13 anos de escolaridade, $40 \%$ eram casados e $87 \%$ estavam empregados.

A Tabela 1 mostra o consumo de energia e nutrientes obtidos pelos dois QFAs e pela média dos três IR24hs. O consumo de energia e da maioria dos nutrientes, tanto pelo QFAl como pelo QFA2, superestimou a ingestão proveniente da média dos três IR24hs. O teste t pareado entre o QFA1 e a média dos três IR24hs mostrou diferenças estatisticamente significativas para a ingestão de energia, proteína, lipídios, carboidratos e cálcio $(p<0,05)$. Já para o QFA2, a diferença só foi significativa para o cálcio.

Ao estratificar a amostra por sexo, observouse que os homens apresentaram médias de ingestão maiores do que as mulheres, tanto para energia como para os nutrientes, com exceção da vitamina A no IR24h (dados não apresentados em tabela).

Os dados de reprodutibilidade, contendo os ICC, estão descritos na Tabela 2. Observa-se que todos os coeficientes demonstraram forte correlação entre o QFA1 e o QFA2. Os melhores resultados foram vistos para vitamina $\mathrm{C}(0,84$; 
Média e desvio-padrão (DP) da ingestão diária de energia e nutrientes avaliados por meio do Questionário de Freqüência Alimentar 1 (QFA1), Questionário de Freqüência Alimentar 2 (QFA2) e dos três inquéritos recordatórios de 24 horas (IR24hs), e édia e DP das diferenças entre os métodos ( $n=83$ ).

\begin{tabular}{|c|c|c|c|}
\hline Nutrientes & $\begin{array}{c}\text { QFA1 } \\
\text { Média (DP) }\end{array}$ & $\begin{array}{c}\text { IR24hs } \\
\text { Média (DP) }\end{array}$ & $\begin{array}{c}\text { Diferença QFA1-IR24hs } \\
\text { Média (DP) }\end{array}$ \\
\hline Energia (Kcal) & $2.354,96(877,64)$ * & $1.988,64(669,07)$ & $366,32(819,55)$ \\
\hline Proteína (g) & $108,09(49,54)$ * & $94,59(39,17)$ & $13,49(42,46)$ \\
\hline Lipídio (g) & $71,09(30,96)$ & $70,91(32,76)$ & $0,18(37,75)$ \\
\hline Carboidrato (g) & $321,97(133,03)$ * & $241,16(84,50)$ & $80,82(108,88)$ \\
\hline Cálcio (mg) & $934,87(440,53)$ * & $666,95(263,37)$ & $267,92(437,29)$ \\
\hline Vitamina A (ER) & $1.393,56(859,57)$ & $760(543,58)$ & $633,10(920,53)$ \\
\hline Vitamina C (mg) & $252,07(208,29)$ & $256,62(713,16)$ & $-4,55(713,52)$ \\
\hline \multirow[t]{2}{*}{ Nutrientes } & QFA2 & IR24hs & Diferença QFA2-IR24hs \\
\hline & Média (DP) & Média (DP) & Média (DP) \\
\hline Energia (Kcal) & $2.026,58(789,21)$ * & $1.988,64(669,07)$ & $37,94(689,99)$ \\
\hline Proteína (g) & $102,03(49,45)$ & $94,59(39,17)$ & $7,44(39,96)$ \\
\hline Lipídio (g) & $63,77(32,85)$ & $70,91(32,76)$ & $-7,14(35,98)$ \\
\hline Carboidrato (g) & $259,44(100,96)$ & $241,16(84,50)$ & $18,29(89,86)$ \\
\hline Cálcio (mg) & $749,18(330,39)$ * & $666,95(263,37)$ & $82,23(326,95)$ \\
\hline Vitamina A (ER) & $1.308,29(1100,86)$ & $760(543,58)$ & $547,81(1038,54)$ \\
\hline Vitamina C (mg) & $196,90(152,89)$ & $256,62(713,16)$ & $-59,71(703,71)$ \\
\hline
\end{tabular}

ER: equivalentes de retinol.

* $\mathrm{p} \leq$ 0,05 para o teste t pareado para as diferenças entre cada QFA e a média dos três IR24hs.

IC95\%: 0,74-0,90), proteína (0,82; IC95\%: 0,72$0,88)$ e energia total $(0,81$; IC95\%: 0,63-0,90). Após o ajuste para energia, somente vitamina C (0,83; IC95\%: 0,72-0,90) e vitamina A $(0,75$; IC95\%: 0,59-0,84) mantiveram coeficientes acima de 0,70 . Destaca-se que o ICC, além de avaliar a associação entre os QFAs, mede o grau de concordância dos valores de ingestão.

Quanto à validade dos QFAs relativa à média de ingestão dos três IR24hs, verificou-se que os ICC não ajustados apresentaram os menores valores para vitamina $\mathrm{A}$ e os maiores valores para proteína, respectivamente, 0,17 e 0,65, no QFA1, e 0,38 e 0,78, no QFA2. Após o ajuste pela energia, com exceção da vitamina C no QFA2, houve redução nos coeficientes para todos os nutrientes. Por outro lado, com a correção para o erro aleatório intrapessoal observou-se aumento nos coeficientes ajustados, em ambos os questionários, com correlações maiores do que 0,4 , exceto para carboidrato, cálcio e vitamina A no QFAl (Tabela 3).

A Tabela 4 mostra que a concordância exata (classificação no mesmo quarto pelos dois métodos) foi de $36 \%$ no QFA1 e $37 \%$ no QFA2, com diferença na amplitude dos percentuais de concordância, respectivamente, 18 (28\% a 46\%) para o QFAl e 5 (35\% a 40\%) para o QFA2. O menor percentual de concordância, em ambos os QFAs, foi visto para a vitamina A. O percentual médio de indivíduos classificados em quartos opostos foi 7\% no QFA1 e 4\% quando se analisou o QFA2. Os valores do índice Kappa ponderado variaram de 0,10 (IC95\%: 0,00-0,26), para o cálcio, a 0,38 (IC95\%: 0,23-0,53), para a vitamina C, no QFA1, e de 0,23 para lipídeo (IC95\%: 0,07-0,38), cálcio (IC95\%: 0,06-0,39) e vitamina A (IC95\%: 0,07-0,38) a 0,38 para energia (IC95\%: 0,24-0,52), no QFA2.

\section{Discussão}

Neste estudo, mediu-se a reprodutibilidade e a validade de um QFA composto por 127 itens alimentares para uso em população adulta da Região Metropolitana de Porto Alegre. Na amostra, teve-se o cuidado de não incluir pessoas com doenças que pudessem interferir no hábito alimentar, que recebiam dieta orientada por profissional da saúde ou que estivessem grávidas. 
Tabela 2

Coeficientes de correlação Intraclasse (ICC) * entre Questionário de Freqüência Alimentar 1 (QFA1) e Questionário de Freqüência Alimentar 2 (QFA2) (n = 83).

\begin{tabular}{|c|c|c|c|c|}
\hline Nutriente & ICC & IC95\% & ICC corrigidos para calorias & IC95\% \\
\hline Energia (Kcal) & 0,81 & $0,63-0,90$ & & \\
\hline Proteína (g) & 0,82 & $0,72-0,88$ & 0,67 & $0,29-0,83$ \\
\hline Lipídio (g) & 0,80 & $0,68-0,87$ & 0,69 & $0,30-0,84$ \\
\hline Carboidrato (g) & 0,79 & $0,47-0,90$ & 0,44 & $-0,19-0,76$ \\
\hline Cálcio (mg) & 0,73 & $0,49-0,84$ & 0,59 & $-0,02-0,81$ \\
\hline Vitamina A (ER) & 0,75 & $0,60-0,84$ & 0,75 & $0,59-0,84$ \\
\hline Vitamina C (mg) & 0,84 & $0,74-0,90$ & 0,83 & $0,72-0,90$ \\
\hline
\end{tabular}

IC95\%: intervalos de 95\% de confiança; ER: equivalntes da retinol.

* Para todas as análises de correlação foram utilizados valores dos nutrientes log transformados.

Tabela 3

Coeficientes de correlação Intraclasse (ICC) entre a média dos três inquéritos recordatórios de 24 horas (IR24hs) e cada um dos Questionários de Freqüência Alimentar (QFA 1 e QFA 2) $(n=83)$.

\begin{tabular}{|c|c|c|c|c|c|c|}
\hline \multirow[t]{2}{*}{ Nutriente * } & \multicolumn{6}{|c|}{ QFA 1} \\
\hline & Bruto & IC95\% & Ajustado ** & IC95\% & 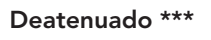 & IC95\% \\
\hline Energia (Kcal) \# & 0,58 & $0,30-0,74$ & - & - & 0,73 & $0,38-0,93$ \\
\hline Proteínas (g) & 0,65 & $0,42-0,78$ & 0,46 & $-0,08-0,71$ & 0,64 & $-0,11-0,99$ \\
\hline Lipídios (g) & 0,57 & $0,33-0,72$ & 0,56 & $0,14-0,76$ & 0,86 & $0,21-1,16$ \\
\hline Carboidrato (g) & 0,56 & $0,09-0,77$ & 0,32 & $-0,19-0,66$ & 0,39 & $-0,23-0,81$ \\
\hline Cálcio (mg) & 0,39 & $-0,03-0,63$ & 0,17 & $-0,14-0,42$ & 0,29 & $-0,24-0,72$ \\
\hline Vitamina A (ER) & 0,17 & $-0,14-0,41$ & 0,15 & $-0,14-0,39$ & 0,23 & $-0,22-0,61$ \\
\hline Vitamina C (mg) & 0,43 & $-0,10-0,69$ & 0,42 & $-0,13-0,69$ & 0,63 & $-0,19-1,03$ \\
\hline \multirow[t]{2}{*}{ Nutriente * } & \multicolumn{6}{|c|}{ QFA 2} \\
\hline & Bruto & IC95\% & Ajustado ** & IC95\% & Deatenuado *** & IC95\% \\
\hline Energia (Kcal) \# & 0,76 & $0,63-0,84$ & - & - & 0,95 & $0,79-1,05$ \\
\hline Proteínas (g) & 0,78 & $0,66-0,86$ & 0,63 & $0,43-0,76$ & 0,88 & $0,60-1,06$ \\
\hline Lipídios (g) & 0,69 & $0,53-0,80$ & 0,53 & $0,27-0,70$ & 0,81 & $0,41-1,07$ \\
\hline Carboidrato (g) & 0,71 & $0,56-0,81$ & 0,61 & $0,39-0,75$ & 0,75 & $0,48-0,92$ \\
\hline Cálcio (mg) & 0,60 & $0,37-0,75$ & 0,42 & $0,12-0,62$ & 0,72 & $0,21-1,06$ \\
\hline Vitamina A (ER) & 0,38 & $-0,07-0,64$ & 0,32 & $-0,10-0,58$ & 0,50 & $-0,16-0,91$ \\
\hline Vitamina C (mg) & 0,49 & $-0,02-0,72$ & 0,50 & $-0,02-0,74$ & 0,75 & $-0,03-1,1$ \\
\hline
\end{tabular}

IC95\%: intervalos de 95\% de confiança; ER: equivalentes de retinol

* Nutrientes log transformados;

** Nutrientes ajustados para energia pelo método dos resíduos;

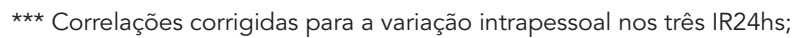

\# Somente deatenuado.

Com isso, evitou-se a possibilidade de alterações no hábito alimentar durante as etapas do estudo. Tendo em vista que o nível de escolaridade pode influenciar nos resultados de validação, optou-se por incluir na amostra pessoas com escolaridade mínima de quatro anos de estudo completos 16
Outro cuidado metodológico foi a obtenção de um IR24h referente ao domingo, uma vez que a ingestão alimentar neste dia costuma ser significativamente maior do que nos outros dias da semana 17 . 
Classificação dos participantes (\%) por quartos de energia e nutrientes dos Questionários de Freqüência Alimentar (QFAs) e da média dos três inquéritos recordatórios de $24 \mathrm{~h}$ (IR24hs) e valores da estatística kappa ponderado com o respectivo intervalo de $95 \%$ de confiança (IC $95 \%)$

\begin{tabular}{|c|c|c|c|c|}
\hline & $\begin{array}{l}\text { Classificação no } \\
\text { mesmo quarto }\end{array}$ & $\begin{array}{l}\text { Classificação em } \\
\text { quartos opostos }\end{array}$ & Kappa & IC95\% \\
\hline \multicolumn{5}{|l|}{ QFA1 } \\
\hline \multicolumn{5}{|l|}{ Nutrientes * } \\
\hline 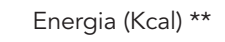 & 39,0 & 8,0 & 0,24 & $0,08-0,41$ \\
\hline Proteína (g) & 32,0 & 6,0 & 0,23 & $0,07-0,38$ \\
\hline Lipídio (g) & 43,0 & 4,0 & 0,34 & $0,19-0,49$ \\
\hline Carboidrato (g) & 37,0 & 5,0 & 0,30 & $0,15-0,45$ \\
\hline Cálcio (mg) & 30,0 & 10,0 & 0,10 & $0,00-0,26$ \\
\hline Vitamina A (ER) & 28,0 & 8,0 & 0,16 & $0,00-0,31$ \\
\hline Vitamina C (mg) & 46,0 & 5,0 & 0.38 & $0,23-0,53$ \\
\hline Média & 36,0 & 7,0 & - & - \\
\hline \multicolumn{5}{|l|}{ QFA2 } \\
\hline \multicolumn{5}{|l|}{ Nutrientes * } \\
\hline 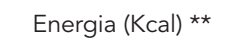 & 40,0 & 1,0 & 0.38 & $0,24-0,52$ \\
\hline Proteína (g) & 37,0 & 2,0 & 0,26 & $0,11-0,42$ \\
\hline Lipídio (g) & 36,0 & 4,0 & 0,23 & $0,07-0,38$ \\
\hline Carboidrato (g) & 36,0 & 8,0 & 0,24 & $0,08-0,40$ \\
\hline Cálcio (mg) & 38,0 & 7,0 & 0,23 & $0,06-0,39$ \\
\hline Vitamina A (ER) & 35,0 & 5,0 & 0,23 & $0,07-0,38$ \\
\hline Vitamina C (mg) & 35,0 & 4,0 & 0,30 & $0,16-0,45$ \\
\hline Média & 37,0 & 4,0 & - & - \\
\hline
\end{tabular}

ER: equivalentes de retinol.

* Nutrientes log transformados e corrigidos para calorias;

** Somente log transformado.

As médias de ingestão de energia e da maioria dos nutrientes foram maiores nos QFAs do que a média de ingestão dos três IR24hs, resultados também encontrados por Ribeiro et al. 18. Estudo realizado com adultos jamaicanos mostrou valores médios mais altos ( $12 \%$ a $61 \%)$ de ingestão absoluta em 10 dos 14 nutrientes analisados no QFA, quando comparados à média de doze IR24hs 19. Em uma pesquisa realizada no Sul do Brasil, o QFA produziu estimativas médias de ingestão absoluta mais altas do que o IR24h para as vitaminas A e C, entre outros nutrientes 20 , achado consistente com o nosso estudo. Ávila et al. 21, também encontraram médias de ingestão absoluta mais altas nos QFAs do que no IR24h, principalmente para vitamina A. No estudo realizado por Slater et al. 7, com adolescentes, não se observaram diferenças entre as médias dos IR24hs e o QFA para energia, lipídeo, vitamina C e cálcio; porém, detectou-se que o QFA superestimou os valores do carboidrato e fibra, e subestimou aqueles para proteí- na, colesterol, ácido graxo polinsaturado e ferro. A superestimação vista para o QFA, no presente estudo, poderia ser atribuída a características inerentes ao instrumento, como o tamanho da lista de alimentos e a sessão para obtenção da freqüência de consumo ${ }^{3}$.

Ao se estratificar por sexo, os resultados apontaram maiores médias de ingestão para os homens, tanto no QFA1 como no QFA2, resultado esperado e coerente com outros estudos 22,23.

Quanto à reprodutibilidade do QFA, a literatura prevê coeficientes de correlação variando de 0,40 a 0,70 4,5,17. Em nosso estudo, os coeficientes de correlação para energia e nutrientes não corrigidos foram sempre maiores do que $0,70 \mathrm{e}$, embora o ajuste para energia tenha reduzido as correlações, os valores ainda ficaram dentro de uma faixa aceitável, com o menor valor para o carboidrato (0,44; IC95\%: $-0,19-0,76)$ e o maior para a vitamina C $(0,83$; IC $95 \%$ : 0,72-0,90).

Um aspecto importante que influencia a reprodutibilidade é o tempo transcorrido entre as 
aplicações dos dois QFAs. Se o intervalo é muito curto, a reprodutibilidade poderia ser superestimada, uma vez que o participante lembraria das respostas dadas ao primeiro questionário. Por outro lado, intervalos muito longos reduziriam as correlações como conseqüência de uma mudança real no padrão da dieta 5 . O tempo médio (um mês) entre a aplicação dos dois QFAs, no presente estudo, pode ser considerado suficiente, tanto para evitar mudanças reais na dieta, como para evitar que os participantes lembrassem das suas respostas dadas anteriormente 5 .

$\mathrm{Na}$ análise estratificada por sexo, os coeficientes de correlação intraclasse, ajustados pela energia, foram maiores entre homens, para a maioria dos nutrientes.

Observou-se, ainda, que as correlações para a reprodutibilidade foram maiores do que os valores de correlação para a validade, consistente com outros estudos 19.

Quanto à validade relativa do QFA, os valores encontrados para os ICC não ajustados são comparáveis ou até maiores do que os dados encontrados na literatura. O ajuste pela energia, entretanto, reduziu os valores dos coeficientes, como encontrado em outros estudos 19,20,21. Segundo Willett 3, o ajuste pela energia aumenta o coeficiente de correlação quando a variabilidade no consumo do nutriente está relacionada com a ingestão energética, porém, diminui quando a variabilidade do nutriente se deve a erros sistemáticos de sub ou super relato do consumo alimentar. Tendo em vista os resultados deste estudo, não se pode descartar que esses erros estejam presentes nos relatos de ambos os métodos.

Uma vez que o erro aleatório intra-sujeito na mensuração de variáveis que estão sendo comparadas tende a reduzir os coeficientes de correlação para zero, a variação dia-a-dia na ingestão alimentar intra-sujeito nos três IR24hs foi corrigida 8 , o que resultou na deatenuação dos coeficientes de correlação ajustados pela energia, entre os QFAs e o método de referência, com valores bem mais altos para o QFA2.

Apesar da correção para a variabilidade intrapessoal nos recordatórios, os valores deatenuados abaixo de 0,4 para carboidrato, cálcio e vitamina A, no QFA1, e intervalos de confiança de grande amplitude, em ambos os questionários, poderiam ser explicados pela variabilidade da ingestão dietética individual, o que aponta para a necessidade de mais replicatas do método de referência, a fim de capturar com mais precisão a ingestão de alguns nutrientes 24 .

Em estudos epidemiológicos, a ingestão de nutrientes é freqüentemente categorizada, de modo a permitir o cálculo de medidas de asso- ciação. Dessa forma, a habilidade de um questionário classificar corretamente os indivíduos segundo o seu nível de ingestão contribui para a obtenção de estimativas de risco corretas. Ao investigar a concordância exata no mesmo quarto, $36 \%$ dos participantes, em média, foram classificados corretamente para o QFA1 e 37\% para o QFA2. Os valores encontrados por Shu et al. 25 para a concordância entre o QFA e o método de referência, variaram de $33 \%$ a 50\%. Em um estudo com amostra de nutricionistas de origem japonesa, 36\% foram classificadas no mesmo quarto pelo QFA e o teste de referência 26 . Como a classificação dos indivíduos segundo as categorias de ingestão dos dois métodos pode agrupar pessoas com níveis de ingestão muito diferentes na mesma categoria, e aqueles com níveis muito semelhantes em categorias opostas, se estiverem muito próximos do ponto de corte, a concordância entre os métodos pode ficar comprometida, particularmente em estudos com pequeno tamanho de amostra 27. Os valores encontrados para o índice kappa foram fracos, porém, concordam com a classificação nos quartos. Em geral, valores maiores correspondem a maior percentual de classificação no mesmo quarto e menor percentual em quartos opostos (vitamina A e lipídeo no QFA 1; energia, proteína e vitamina C no QFA2).

No presente estudo, observou-se maior amplitude na distribuição dos valores do percentual de concordância para o QFA1 do que para o QFA2, respectivamente, 18 e 5. Também a média de classificação em quartos opostos foi maior no QFA1 do que no QFA2.

Como se pode observar, nossos achados apontam para maior precisão nas informações obtidas pelo QFA aplicado no segundo momento da pesquisa (QFA2). Com isso, levantam-se duas possibilidades. A primeira explicação seria que, ao responder ao QFA2, o entrevistado estava mais preparado, pois já conhecia o instrumento e sua forma de aplicação, tornando-se mais apto em quantificar as suas porções 28 . Outra possibilidade para esses achados seria a ocorrência do efeito Hawthorne, o qual significa que, no período entre as entrevistas, as pessoas teriam alterado sua alimentação por estarem participando da pesquisa.

Entre as limitações deste estudo poderíamos citar o uso do IR24h como método de referência para validar o QFA, pois as fontes de erro podem estar correlacionadas, já que ambos os métodos dependem da memória 7. Entretanto, considerando-se que os intervalos para lembrar o consumo alimentar foram diferentes, acredita-se que os erros tenham sido minimizados. A aplicação de apenas três IR24hs também pode ter sido uma 
limitação para a mensuração da ingestão habitual da maior parte dos micronutrientes ${ }^{3}$.

É possível, também, que o período de referência para o QFA (um mês) não tenha sido suficiente para contemplar o padrão da dieta habitual dos participantes do estudo, o que poderia resultar em correlações mais baixas do que normalmente encontramos na literatura para alguns nutrientes, como foi o caso do cálcio, neste estudo. Contudo, dados de uma metanálise de quarenta estudos de validação de QFA, com diferentes tempos de referência, mostraram que as correlações para esse nutriente variaram de 0,20 a 0,75 29 .

Outra limitação inclui o uso de lista de alimentos derivada de pesquisa desenvolvida em Niterói. Entretanto, considera-se que a diferença nos padrões alimentares das regiões Sul e Sudeste não seja tão marcante. Além disso, o QFA previa espaço para o registro de alimentos não contemplados pela lista original. Vale destacar, também, que as correlações encontradas para o estudo de validade são consistentes com dados da literatura, indicando que os alimentos consumidos em
Niterói podem ser utilizados para compor a lista do QFA para o Sul do Brasil.

Por ser uma amostra com voluntários, não se pode descartar o viés do voluntariado, o que poderia contribuir para a obtenção de correlações mais fortes do que na população geral, já que os voluntários podem ter motivação diferenciada para responder a múltiplos questionários 30 .

Em conclusão, o QFA apresentou reprodutibilidade satisfatória para todos os nutrientes, com exceção do carboidrato, e validade relativa razoável, especialmente para os macronutrientes e vitamina $\mathrm{C}$, quando se considerou o QFA1, e validade de moderada a alta para o QFA2, entre adultos com escolaridade superior a quatro anos de estudo, residentes na região metropolitana do sul do Brasil. Com base nos achados, há indícios de que proporcionar uma prévia apresentação e explicação mais detalhada do instrumento, ou seja, do QFA a ser utilizado para medir a ingestão, poderia levar a melhores estimativas de ingestão alimentar; por outro lado, ainda não se conhece a magnitude de um possível viés de informação com esta abordagem prévia.

\section{Resumo}

O estudo avaliou a reprodutibilidade e a validade relativa de um questionário de freqüência alimentar (QFA) em 83 indivíduos adultos, do Sul do Brasil. Utilizou-se o coeficiente de correlação Intraclasse (ICC), classificação em quartos de ingestão pelo QFA e IR24h e indice Kappa ponderado. Os nutrientes foram log transformados e corrigidos para calorias. A atenuação nas correlações foi corrigida pela variabilidade intrapessoal. Para a reprodutibilidade, os valores das correlações variaram de 0,44 (IC95\%: -0,19-0,76) a 0,83 (IC95\%: 0,72-0,90) para carboidrato e vitamina C, respectivamente. No estudo de validade, os coeficientes deatenuados variaram de 0,23 (IC95\%: -0,22-0,61) a 0,86 (IC95\%: 0,21-1,16) para a vitamina A e lipídeos, no QFA1, e de 0,50 (IC95\%: -016-0,91) a 0,95 (IC95\%: 0,79-1,05) para vitamina A e energia, no QFA2. O índice Kappa ponderado apresentou valor mais baixo para o cálcio $(0,10$; IC95\%: 0,00-0,26) e valores mais altos para vitamina C no QFA1 (0,38; IC95\%: 0,23-0,53) $e$ energia no QFA2 (0,38; IC95\%: 0,24-0,52). Os resultados indicam reprodutibilidade satisfatória para todos os nutrientes, com exceção do carboidrato, e validade relativa razoável, especialmente para os macronutrientes e vitamina $C$.

Questionários; Comportamento Alimentar; Adulto

\section{Colaboradores}

M. T. A. Olinto e L. A. Anjos estiveram envolvidos em todas as etapas do projeto. R. L. Henn colaborou nas análises dos dados e na redação do manuscrito. V. Wahrlich colaborou na análise dos dados e na discussão do artigo. A. F. Zanolla supervisionou o trabalho de campo, analisou os dados e redigiu este manuscrito, que foi revisado e aprovado pelos demais autores.

\section{Agradecimentos}

O projeto teve apoio financeiro da Coodenação de aperfeiçoamento de Pessoal de Nível superior (CAPES) dentro do Edital PROCAD 01/2005, no. 0257052. L. A. Anjos e M. T. A. Olinto receberam bolsa de produtividade em pesquisa do Conselho Nacional de Desenvolvimento Científico e Tecnológico (CNPq, processo 311801/200). 


\section{Referências}

1. World Health Organization. Reducing risks, promoting healthy life. World Health Report. Geneva: World Health Organization; 2002.

2. World Health Organization. Diet, nutrition and the prevention of chronic diseases. Report of a Joint FAO/WHO/UNU Expert Consultation. Geneva: World Health Organization; 2003. (WHO Technical Report Series, 916).

3. Willett W. Nutritional epidemiology. Oxford: Oxford University Press; 1998.

4. Thompson F, Byers T. Dietary assessment resource manual. J Nutr 1994; 124:2245S-317S.

5. Cade J, Thompson R, Burley V, Warm D. Development, validation and utilisation of food-frequency questionnaires - a review. Public Health Nutr 2002; 5:567-87.

6. Lopes A, Caiaffa W, Mingoti S, Lima M. Ingestão alimentar em estudos epidemiológicos. Rev Bras Epidemiol 2003; 6:209-19.

7. Slater B, Philippi S, Marchioni D. Validação de Questionários de Freqüência Alimentar - QFA: considerações metodológicas. Rev Bras Epidemiol 2003; 6:200-8.

8. Rosner B, Willett W. Interval estimates for correlation coefficients corrected for within-person variation: implications for study design and hypothesis testing. Am J Epidemiol 1988; 127:377-86.

9. Lee J, Kolonel L, Hankin J. On establishing the interchangeability of different dietary-intake assessment methods used in studies of diet and cancer. Nutr Cancer 1989; 5:215-8.

10. Bossan FM, Anjos LA, Vasconcellos MTL, Wahrlich V. Nutritional status of the adult population in $\mathrm{Ni}$ terói, Rio de Janeiro, Brazil: the Nutrition, Physical Activity, and Health Survey. Cad Saúde Pública 2007; 23:1867-76.

11. Zaboto C. Registro fotográfico para inquéritos dietéticos: utensílios e porções. São Paulo: Editora Unicamp; 1996.

12. Núcleo de Estudos e Pesquisas em Alimentação. Tabela de composição de alimentos, versão 2 . Campinas: Universidade Estadual de Campinas; 2006.

13. Pinheiro A, Lacerda E, Benzecry E, Gomes M, Costa V. Tabela para avaliação de consumo alimentar em medidas caseiras. 5a Ed. Rio de Janeiro: Atheneu; 2005.

14. Willett W, Stampfer M. Total energy intake: implications for epidemiologic analyses. Am J Epidemiol 1986; 124:17-27.

15. Willett W. Future directions in the development of food-frequency questionnaires. Am J Clin Nutr 1994; 59:171-4.

16. Kristal AR, Reng Z, Coates RJ, Oberman A, George V. Associations of race/ethnicity, education and dietary intervention with the validity and reliability of a food frequency questionnaire. Am J Epidemiol 1997; 146:856-69.

17. Beaton G, Burema J, Ritenbaugh C. Errors in the interpretation of dietary assessments. Am J Clin Nutr 1994; 65:1100S-7S.
18. Ribeiro A, Sávio K, Rodrigues M, Costa T, Schivitz B. Validação de um questionário de freqüência de consumo alimentar para população adulta. Rev Nutr 2006; 19:553-62.

19. Jackson M. Reproducibility and validity of a quantitative food-frequency questionnaire among Jamaicans of African origin. Public Health Nutr 2001; 4:971-80.

20. Henn RL. Padrão alimentar em uma população adulta da Cidade de Porto Alegre, RS, 2005 [Tese de Doutorado]. Porto Alegre: Universidade Federal do Rio Grande do Sul; 2006.

21. Ávila M, Romieu I, Parra S, Ávila J, Madrigal H, Willett W. Validity and reproducibility of a food frequency questionnaire to assess dietary intake of women living in Mexico City. Salud Pública Méx 1998; 39:133-40.

22. Shimizu H, Ohwaki A, Yoko K, Takatsuka N, Ido M, Kawakami N, et al. Validity and reproducibility of a quantitative food frequency questionnaire for a cohort study in Japan. Jpn J Clin Oncol 1998; 29:38-44.

23. Hebert J, Gupta P, Bhonsle R, Sinor P, Mehta H, Mehta F. Development and testing of a quantitative food frequency questionnaire for use in $\mathrm{Gu}$ jarat, India. Public Health Nutr 1999; 2:39-50.

24. Eck L, Klesges L, Klesges R. Precision and estimated accuracy of two short-term food frequency questionnaires compared with recalls and records. J Clin Epidemiol 1996; 9:1195-200.

25. Shu X, Yang G, Jin F, Liu D, Kushi L, Wen W, et al. Validity and reproducibility of the food frequency questionnaire used in the Shanghai women's health study. Eur J Clin Nutr 2004; 58:17-23.

26. Cardoso M, Kida A, Tomita L, Stocco P. Reproducibility and validity of a food frequency questionnaire among women of Japanese ancestry living in Brazil. Nutr Res 2001; 21:725-33.

27. Masson LF, McNeill G, Tomany JO, Simpson JA, Peace HS, Wei L, et al. Statistical approaches for assessing the relative validity of a food-frequency questionnaire: use of correlation coefficients and the kappa statistic. Public Health Nutr 2003; 6:313-21.

28. Pietinen P, Hartman A, Haapa E, Rasamen L, Haapakoski J, Palmgren J, et al. Reproducibility and validity of dietary assessment instruments II. A qualitative food frequency questionnaire. Am J Epidemiol 1988; 128:667-76.

29. Molag ML, de Vries JHM, Ocké MC, Dagnelie PC, van den Brandt PA, Jansen MCJF, et al. Design characteristics of food frequency questionnaires in relation to their validity. Am J Epidemiol 2007; 166:1468-78.

30. Fisberg R, Slater B, Marchioni D, Martini L. Inquéritos alimentares: métodos e bases científicos. São Paulo: Editora Manole; 2005.

Recebido em 29/Abr/2008

Versão final reapresentada em 09/Out/2008

Aprovado em 29/Out/2008 